\title{
Algunos elementos teóricos de las políticas públicas: análisis para su formulación*
}

\author{
Rincón Becerra, María T.**
}

\section{Resumen}

El presente trabajo tiene como objetivo realizar una reflexión teórica sobre el tema de las políticas públicas, haciendo especial referencia a la fase de formulación de la política pública y como parte de ella a las etapas de identificación de un problema y formulación de la agenda; así como la formulación de soluciones y toma de decisiones, caracterizada cada una de estas etapas siguiendo los modelos decisionistas y otros enfoques. Se adelanta una investigación descriptiva y documental. Los resultados dan cuenta que: 1) los modelos decisionistas fueron propuestos para realidades como la norteamericana, tal y como se evidencia en la literatura relacionada con el tema en cuestión; 2) que existen otros enfoques que emanan de otras realidades, como la latinoamericana que permiten visualizar otra orientación en el proceso de formulación de las políticas públicas. Se concluye que para el estudio de los procesos de formulación de la política pública, se hace necesario considerar diversos modelos teóricos, en los cuales se conjugue el papel del Estado y su relación con la sociedad, de tal manera de establecer un análisis objetivo y equilibrado, sin perder de vista las limitaciones que puede tener cada modelo.

Palabras clave: Formulación de políticas públicas, contenido de las políticas; modelos de decisión.

Recibido: 30-10-13. Aceptado: 15-05-14

* $\quad$ Este trabajo forma parte de los resultados del proyecto de Investigación Información para la gestión pública, financiado el Consejo de Desarrollo Científico y Humanístico (Condes) y adscrito al Centro de Estudios de la Empresa (CEE) de la Facultad de Ciencias Económicas y Sociales (FCES) de La Universidad del Zulia (LUZ).

** Lic. en Ciencia Política. Magíster en Dirección y Gestión Pública Local. Dra. En Ciencias Sociales, Mención Gerencia. Docente e Investigadora del CEE de FCES de LUZ. Acreditada en el Programa de Estímulo a la Investigación e Innovación, Nivel B (PEII). E-Mail: materinbe2@yahoo.com / materinbe@hotmail.com 


\section{Some Theoretical Elements of Public Policies: Analysis for their Formulation}

\section{Abstract}

The objective of this study is to make a theoretical reflection on the topic of public policies, with special reference to the phase of their formulation and, as part of this, to the stages of identifying a problem and formulating the agenda, as well as formulating solutions and making decisions. Each of these stages will be characterized according to decisionist models and other approaches. The research is descriptive and documentary. Results indicate that: 1 ) decisionist models were proposed for realities like that of North America, as shown in the literature related to the topic in question; 2) other approaches exist that emanate from other realities, such as Latin America, that permit visualizing another orientation in the process of formulating public policies. Conclusions are that, to study the processes of formulating public policy, it is necessary to consider diverse theoretical models, in which the role of the State is combined with its relation to society, so that an objective, balanced analysis is established without losing sight of the limitations each model may have.

Key words: Public policy formulation, policy content, decision models.

\section{Introducción}

Existen dos posturas frente al análisis de las políticas públicas, una orientada hacia el análisis de la política pública; y la otra hacia el análisis para la política pública. En la primera, se ubica: 1) Análisis de determinación de políticas públicas: este se ocupa del qué, él para quién, el cómo y el cuándo; 2) Análisis del contenido de las políticas públicas: consistente en la descripción del fondo de la política y su proceso; y (en esta se erige la política); y, 3) Seguimiento y evaluación de las políticas públicas: que examina el desempeño que la política como tal pueda tener. En cambio la segunda postura, inherente al análisis para la política, se encuentran tres aristas para la búsqueda de: 1) Argumentos para realizar el monitoreo y evaluación de la política; 2) Información para la política; es decir, elaboración de argumentación para ejercer influencia en la agenda de políticas; y 3) Análisis a favor de una causa; es decir, hacia la búsqueda de contrarrestar una anomalía de política y poder mejorarla (Roth, 2007).

La identificación de los elementos teóricos; están dirigidos hacia el proceso de formulación y el contenido de la política, como ejes reveladores de la política formal, dado que aún cuando existe abundancia de textos inherentes a la materia entre los que destacan: Grau (2002); Subirats (1989); Subirats y Gomá (1998); Álvarez (1992); Muller (2006); Dunn (1994); Urdaneta (2010); Kingdon (1984); Aguilar (1994); Lindblom (1994); Arellano (1996); Klijn (1998); Lindblom (1994); Roth (2004 y 2007); Etzioni (1994); Aktouf (1998); Simon (1986); Chaqués (2004); Fleury (2002); Zurbriggen (2003); Dunn (1994); Dror (1994); y Ochoa (2007), ninguno se detiene en estudiar de manera independiente cada fase de la política, las cuales se constituyen a la vez 
en un proceso; es decir, no son meras etapas de un ciclo, sino que cada una de ellas se construye en un continuo y sucesivo de subfases, donde la formulación de políticas usualmente implica un debate sobre cuáles acciones realmente darían resultados.

Se hace referencia esencial a la fase de formulación de la política pública, aludiendo en ella: 1) identificación del problema y formación de la agenda; y 2) formación de soluciones y toma de decisiones; esta fase se ha construido tradicionalmente bajo la lógica de los modelos decisionistas, como lo han sido la racionalidad absoluta, racionalidad limitada, el incrementalismo y la racionalidad imperfecta.

La investigación que se adelantó fue de tipo documental, entendida esta como“....un proceso basado en la búsqueda, recuperación, análisis, crítica e interpretación de datos secundarios, es decir, los obtenidos y registrados por otros investigadores en fuentes documentales: impresas, audiovisuales o electrónicas. La metodología consistió en la revisión y análisis de documentos teóricos, apoyada en la técnica del arqueo bibliográfico, sobre tópicos tales como: formulación de las políticas públicas, contenido, y modelos de decisión.

\section{Políticas Públicas: generalidades sobre su proceso}

En el análisis de políticas públicas, se encuentra el tradicional enfoque secuencial por etapas de la política, el cual se ha constituido en un modelo teórico metodológico para entender la secuencia de las decisiones de las autoridades gu- bernamentales. Este enfoque tiene sus orígenes en los estudios casuísticos norteamericanos, siendo popularizado por Charles Jones a principios de la década de los setenta, y propuso cinco fases, de acuerdo a los señalamientos de Muller (2006:54) y Grau (2002: 37-45):

a) La identificación del problema y formación de la agenda: en esta se origina la política, es donde algunos aspectos de la realidad pasan a percibirse como problemas que reclaman una atención pública y comienzan a estar en las agendas de acción de los gobiernos y los administradores.

b) Formulación de las soluciones y toma de decisiones: esta fase conlleva a la toma de decisiones por parte de las autoridades públicas, se le da tratamiento formal al problema, se establecen las alternativas de solución y se decide la política que posteriormente será implementada.

c) Implementación: se pone en marcha la política decidida en la fase anterior; se organizan los medios para ponerla en práctica; es decir, interpretar las directrices gubernamentales y aplicarlas.

d) La evaluación: es una fase preliminar que pone en perspectiva los resultados de la política implementada, comprende la medición de datos, análisis y formulación de las recomendaciones; es decir, se da el análisis de los efectos, consecuencias e impactos de la política pública.

e) Mantenimiento, revisión o terminación de la política: es una fase de cierre de la acción o de puesta en marcha de una nueva acción (Muller, 2006. 54). 
Las posturas de Grau (2002) y de Muller, (2006), manifiestan una orientación lineal de las fases de la política pública; sin embargo admiten que ha sido un esquema metodológicamente utilizado para explicar las políticas públicas. Las críticas básicas a este modelo se concentran en la omisión de la dimensión política del proceso; a lo sumo, como lo indica Grau (2006: 48), vale para entender el funcionamiento del sistema político en sus ámbitos de elaboración y definición de políticas públicas.

Subirats (1989), contrariamente a los señalamientos de Grau (2002) y Muller (2006), considera que la política pública recorre un itinerario no estrictamente lineal, con interacciones complejas entre fases; ya que el orden de las etapas puede ser inverso o perturbado, a) una decisión puede ser tomada antes de plantear el problema que se pretende resolver; b) es muy difícil fijar con precisión el momento exacto de la toma de decisión, y separar en la práctica cada una de las etapas; c) la tendencia es a situar la evaluación sólo al final del proceso, ya que en la realidad los observadores o administradores bien sea formal o informalmente van evaluando las fases de las mismas; $y$ d) la terminación de la política es con frecuencia puro ejercicio escolar, pues los problemas políticos nunca son resueltos efectivamente (Muller, 2006; Roth, 2007; Grau, 2002; Urdaneta, 2010).

Por otra parte, Álvarez (1992: 15 y 31 ), propone un enfoque para el análisis de políticas públicas centrado en la dimensión política del proceso, en el que se hace necesario destacar la importancia del análisis del conflicto y el poder. Sugie- re un enfoque de análisis de políticas públicas basado en la optimización, como la fase en la que se halla la solución alternativa más acorde a un problema dado, donde la solución del problema es el objetivo del proceso de la política pública (en el entendido que abarca desde la formulación hasta la evaluación). En la implementación se realizan las acciones que permiten materializar la solución prevista, y en la evaluación se determinan los efectos observables de las acciones emprendidas y se mide la distancia entre los logros alcanzados y el objetivo previsto, lo cual realimenta la fase de optimización de la subsiguiente decisión.

La interacción de las etapas se engloba dentro de lo que el autor denomina el proceso de formación de la política pública. $Y$ diferencia entre formulación y formación de la política, ya que considera que en la formulación se realiza la definición de objetivos y cursos de acción, el cálculo deliberativo para determinar la relación entre medios y fines. En esta etapa el momento crítico del proceso de formulación de políticas es la optimización de los objetivos. Pero sugiere que las Políticas Públicas no son relación de medios fines de ejecución automática. La Formación la asume como una relación compleja y flexible de interconexión de procesos, en la que sus problemas y soluciones son constantemente redefinidos 0 re inventados. La política acepta cambios que deben explicar ¿cómo? y ¿por qué sucede? Lo que implica ser previsivos y determinar evaluaciones realistas.

Asimismo, es importante destacar que el enfoque de las políticas públicas, a pesar de ser un modelo tradicional del análisis, ha contado con un conjunto de críticas, entre ellas destacan: 
- Es un enfoque irreal de las fases del proceso de las políticas públicas al considerarlo a partir de un orden lógico y sucesivo.

- El orden de las etapas puede ser inverso o perturbado, una decisión puede ser tomada antes de plantear el problema que se pretende resolver 0 simplemente la decisión es no considerar una acción.

- Este enfoque lineal no se da tan claramente, la dinámica sociopolítica es más compleja, en algunos casos es cíclica, en otros puede brincarse fases, todo dependiendo del contexto sociopolítico, el juego de poder en el sistema político, la dinámica de los actores y de la aparición de situaciones especiales que modifican el contexto ( $\mathrm{Pe}$ terson, 2003).

- Existe poca relación entre las fases y la realidad objeto de intervención.

- Tiene carácter descriptivo y baja capacidad para explicar causas, consecuencias y variaciones en las políticas.

- Se limita a las élites gubernamentales y a la verticalidad del proceso

- Es una herramienta analítica metodológica y no una teoría del proceso de políticas.

A pesar de las críticas que surgen para el enfoque de las políticas, es importante destacar, que para hacer un análisis realista de las políticas públicas se requiere que en la realidad se dé un cambio de actitud respecto al análisis convencional y normativo; resaltar el rol de las relaciones de poder; los conflictos de interés; la diversidad e incompatibilidad de valores de la sociedad; superar la separación entre administración y política; y romper con la linealidad del proceso político.

Para el análisis de las políticas es necesario, analizar el contenido y construir el proceso de su elaboración, es decir las acciones que conducen a la definición de la agenda y su intento de resolverlo.

\section{Política Pública: su proceso de formulación}

La formulación de la política es una de las fases más trascendentales del proceso de la política pública, pues se considera que es en esta, donde se da origen a la misma. Dos subfases se identifican: a) Identificación del problema y formación de la agenda y, b) elaboración de las alternativas de acción y toma de decisiones.

\subsection{Identificación del problema y formación de la agenda}

En esta subfase "...determinados aspectos de la realidad se convierten o pasan a percibirse como problemas ante los que se reclama una atención pública... y pasan a estar en las agendas de acción de los gobiernos y las administraciones..." (Grau, 2002: 37).

Álvarez (1992), refiere que los problemas son las necesidades de la sociedad y se denominan demandas, las cuales pueden ser manifiestas (a través de marchas, protestas) o latentes (es una necesidad que aún no se ha hecho manifiesta). Estas demandas surgen del seno de la sociedad y tienen posibilidad de acceder a las arenas de confrontación, esta probabilidad depende del poder del grupo que la expresa; en esas arenas se discute 
con relación a su definición, explicación, evaluación o previsión de sus efectos, transformándola en un tema para la agenda política. Para que el problema entre en la agenda pública, intervienen diferentes actores políticos que, según Muller (2006) provendrán del sindicalismo, del mundo de la política, de las asociaciones de ciudadanos o de grupos creados circunstancialmente.

Refiere Grau (2002: 37), que entre los agentes que pueden influir están: "la presencia social, la actividad de los grupos de interés, los acontecimientos catastróficos y de crisis, el cambio de valores, los procesos internacionales, la influencia de las élites políticas, el papel de los técnicos y los expertos...", entre otros. Es decir que pueden ser diversos los agentes o factores que pueden incidir en la identificación de los problemas y su inclusión en la agenda. Por ello, para el estudio de cualquier asunto identificado como un problema, es necesario considerar el elemento contextual en el que se gesta dicho asunto; es decir, el problema que una política quiere resolver implica y exige conocer el entorno socioeconómico y cultural del que forma parte, por el que existe y está determinado, así como el proceso histórico del que es efecto y momento de su tendencia (Aguilar, 2003: 49), puesto que los mismos afectan o determinaran el desenvolvimiento de la acción política a diseñar y por ende a implementar.

Muller (2006) considera que un problema puede tener una posibilidad de ser inscrito en la agenda política sí: a) unas élites (sindicales, administrativas, políticas), unos ciudadanos que pueden estar organizados definen una situación como problemática; b) el descubrimiento del proble- ma puede calificarse como perteneciente a la esfera de competencia de las autoridades públicas; c) se espera la intervención de la sociedad política, incluyendo la opción de no hacer nada; d) que pueda estar constituida por medidas concretas que conforman la sustancia o contenido de una política pública; e) que comprenda decisiones que requieran de asignación de recursos; f) que se inscriba en un marco general de acción; y g) que tenga un público cuya situación se afecte por esa política, los cuales pueden ser pasivos y otros se podrán organizar para influir en la elaboración de los programas políticos.

La formación de la agenda puede verse como el proceso a través del cual ciertas cuestiones tienen el potencial de convertirse en asuntos de política pública. Se contemplan dos tipos de agendas: a) La agenda pública, que "está integrada por todas la cuestiones que los miembros de una comunidad política perciben comúnmente como merecedores de la atención pública" (Aguilar, 2000: 31-32), y b) la agenda política, que se distingue de la anterior por centrarse en las respuestas y propuestas que ofrecen los grupos políticos y las instituciones sociales sobre determinados temas que llaman la atención pública (Aguilar, 2000: 31-32). En la agenda política deberían estar normalmente las demandas más sentidas que surgen de la sociedad. Sin embargo, éstas son muy variadas, por lo que su inserción en la agenda depende de las valoraciones de cada ciudadano, de cada tomador de decisiones y de lo que otras personas esperan de las políticas públicas (Lindblom, 1994).

La agenda política siempre será más acotada que la agenda pública, so- 
bre todo porque los problemas de la sociedad que se convierten en asuntos de políticas públicas se enfrentan a la limitada capacidad de la burocracia gubernamental para diseñar soluciones específicas a cada uno de esos problemas. La dinámica de este proceso provoca que algunos actores incidan más que otros, gracias a su grado de organización y/o los recursos con que se cuentan, y a las condiciones y características del sistema político, en donde se registra un proceso de filtración de las demandas. Entre algunas interrogantes que permutan el análisis, se encuentran:

- Diversas pueden ser las interrogantes que se consideren para la fase de inicio de la política, entre ellas pueden destacar: ¿Hay tiempo para hacer el análisis?, ¿cuál es el asunto o problema a tratar?, ¿Cómo y por qué un asunto o tema se constituye en objeto de atención por parte de las instituciones públicas?, o ¿por qué algunos temas o asuntos no llegan a convertirse en un curso de diseño y posterior acción por parte del gobierno? ¿Cómo interactúan los grupos de interés y de presión para que un tema o asunto sea tratado y otros se queden aletargados en el tiempo?, ¿Hasta qué punto el tema o asunto tiene implicaciones políticas?, ¿Qué influencia ejercen los medios de comunicación para que un asunto público se convierta en un tema o problema de política?, ¿Existen ya posiciones fijas sobre el tema o asunto?, ¿Qué grado de consenso existe sobre el tema y su solución?, ¿Cómo entra un asunto a la agenda?, estas entre otras tantas interrogantes más que pudieran considerase en esta subfase.
Una vez determinado el asunto o problema que entra a formar parte de la agenda, el paso siguiente consiste en establecer los lineamientos que permitirán afrontar el problema objeto de atención y las alternativas de soluciones, para ellos la toma de decisiones se considera desde diversas perspectivas; sin embargo en este trabajo se considera la perspectiva racionalista.

\subsection{Formulación de las soluciones y toma de decisiones}

Es esta subfase es en donde se concreta la toma de decisiones por parte de las autoridades públicas (Grau, 2002). Cuando un asunto entra a formar parte de la agenda, el siguiente paso es establecer cómo se va a solucionar el o los problemas y posteriormente en escoger qué curso de acción se va a llevar a cabo.

En este sentido, puede existir una solución para cada problema, varias soluciones para un problema, una solución para varios problemas, y problemas que no tengan solución o que no requieran ninguna acción inmediata. De esta gama de alternativas de soluciones, es preciso referir que los problemas que son susceptibles de varias soluciones son aquellos que pueden dar paso a la elaboración de la política pública. Para decidir la mejor solución o soluciones es necesario considerar el respaldo ideológico-político; la valoración de los criterios técnicos y administrativos (Ruiz, 1996). Para esta subfase corresponde un conjunto de preguntas que pudieran servir para guiar el análisis

- ¿El proceso de definición de los problemas se convierte en el de búsqueda, creación y primer examen de las ideas 
o vías de solución?, ¿Se estudian las propuestas para ver si son oportunidades reales de mejora, y para comprobar cuál es su beneficio neto? ¿Qué alcance tienen las repercusiones que la solución del tema puede desencadenar? ¿Cuál es la significación de los grupos afectados? ¿Puede el tema tener ramificaciones 0 afectar a otros temas? ¿Puede ser que la actividad que desencadene la resolución del tema afecte en el futuro a la flexibilidad de actuación del mismo servicio? ¿Cómo se toman las decisiones para llevar a cabo una política? ¿Quiénes participan en la toma de decisiones? ¿Cómo es el proceso de elección de una u otra alternativa de solución? ¿Se toman decisiones por inercia administrativa 0 previa discusión entre los actores? ¿incide la política y la administración en el contenido que orientará la o las decisiones?

\subsubsection{Modelos de decisión}

El análisis en el proceso de decisión, se lleva a cabo desde diversas perspectivas, para este caso se considera, la orientación racional, básicamente: la racionalidad absoluta (Etzioni, 1994); racionalidad limitada (Simon, 1986), el incrementalismo (Lindblom, 1994), y al racionalidad imperfecta, tal y como la define Arellano (1996).

\section{a) Racional absoluta}

En el modelo decisional centrado en la racionalidad absoluta ${ }^{1}$, su perspectiva panorámica está arraigada en el pensamiento occidental y encuentra su expresión clásica en el Discurso del Método de Descartes. Este modelo de decisión se abstrae del mundo real al asumir que el decisor se comporta de la siguiente manera: tiene un problema bien definido; una gama completa de alternativas a tomar en consideración; una información básica completa sobre las consecuencias de cada alternativa; sobre los valores y preferencias de los ciudadanos; y del tiempo, la capacidad y los recursos.

La racionalidad absoluta, alude al hecho que "un actor es consciente de un problema, define una meta, sopesa cuidadosamente los medios alternativos $y$ escoge entre ellos de acuerdo con una estimación de sus méritos respectivos con referencia al estado de cosas preferido" (Etzioni, 1994: 266). Este modelo de racionalidad hace mención a un sistema organizado y estable de preferencias y una capacidad que le permite calcular los efectos de su elección para todos los cursos de acción posibles y seleccionar la que le permite un resultado óptimo.

Este modelo de decisión se caracteriza por evidenciar: a) un esquema secuencialmente lineal del proceso de toma de decisiones, que abarca no solo la for-

1 El enfoque racional positivista es la versión extrema de los principios elementales de la razón (Arellano, 1996). 
mulación sino también la implementación de la política; b) un único actor en la toma de decisión; c) un modelo utópico e idealista en el cual no existen los antagonismos ni hay valores culturales que determinen conductas diversas. Los problemas no siempre están bien definidos, no necesariamente se cuenta una gama amplia de alternativas de solución, mucho menos información completa sobre la situación planteada y sobre las consecuencias que pueda generar cada alternativa, además es inadmisible tener claro los valores y preferencias que los ciudadanos pueden tener; es inverosímil tener una amplia disponibilidad de tiempo, el cual es un recurso escaso cuando de decisiones se trata; y los recursos humanos, materiales, financieros, entre otras nunca son suficientes (Etzioni, 1994).

Como es de observar, la racionalidad absoluta, se constituye en un modelo normativo al fundamentarse en un sistema organizado y estable de preferencias y una capacidad que le permite calcular los efectos de su elección para todos los cursos de acción posibles y seleccionar la que permite un resultado óptimo.

\section{b) Modelo de Racionalidad Limitada}

En una posición crítica a la racionalidad absoluta, surge el enfoque de la racionalidad limitada, el cual fue propuesto por Herbert Simon a principios de la década de los ochenta, ellos consideraron que era necesario pensar y actuar sobre los problemas desde otra perspectiva, ya que en la realidad, el decisor se encuentra frente a unas condiciones que limitan sus posibilidades de aplicar el esquema clásico de racionalidad absoluta.
Estos autores destacan que los decisores reales se enfrentan a "problemas ambiguos y mal planteados; información incompleta acerca de las alternativas;... de los fundamentos y antecedentes del problema;... de las consecuencias de las supuestas alternativas; del contenido de los valores, preferencias e intereses, y tiempo, habilidades y recursos limitados" (Forester, 1994: 318). Subirats (1989) señala que en este tipo de modelo de decisión, el decisor puede ser unitario o puede presentarse una coalición de actores, y cuyo criterio de elección es la satisfacción, ya que ningún individuo puede tener todo el conocimiento de los elementos de conjunto de una situación, ni de todas las consecuencias de los actos que pueda emprender, ni de todas las opciones posibles. La decisión se hace en un contexto y en el marco de un proceso que a menudo depende más de las formas habituales de funcionar, que de análisis exhaustivos y racionales. Por tanto, esta elección efectuada desde afuera no puede ser la mejor decisión, sino solo la más satisfactoria en dichas circunstancias, entre varias opciones posibles (Aktouf, 1998).

Esta racionalidad se caracteriza por dos aspectos: búsqueda y satisfacción. Si el tomador de decisiones no conoce las alternativas de inicio, entonces él debe buscarlas mediante un proceso de aproximaciones sucesivas hasta encontrar la más satisfactoria o buena, para la situación dada y el nivel de aspiraciones previamente fijado. Este proceso se realiza con una cantidad razonable de cálculos y utilizando información muy incompleta con mecanismos de adaptación y aprendizaje, siguiendo criterios y reglas relativamente sencillos y manejables que no exigen im- 
posibles a su capacidad de pensamiento. El modelo de racionalidad limitada es más rico que el modelo de elección racional porque no sólo trata del equilibrio, sino también de la manera de alcanzarlo. También se denomina modelo de racionalidad procesal porque es el resultado de una deliberación apropiada, de un proceso de razonamiento (Simon, 1986).

Este modelo trata el equilibrio y la manera de alcanzarlo; la existencia de un hombre administrativo con necesidad de buscar alternativas de decisión, la sustitución de resultados máximos u óptimos por soluciones satisfactorias y el uso de mecanismos de aprendizaje y adaptación utilizando la información disponible. El modelo de racionalidad limitada, sugiere que el mismo se basa en observaciones de los procesos de decisión reales de las organizaciones. Tales observaciones indican que los individuos modifican o hasta ignoran el modelo racional, especialmente frente a ciertos tipos de decisiones adaptativas e innovadoras.

Un decisor tiene enormes limitaciones en su capacidad de tomar en cuenta, para su decisión, todos los hechos que se dan en el mundo que lo circunda, que serían relevantes para esa decisión. Estos límites surgen simplemente porque los actores tienen un conocimiento restringido, tienen capacidades limitadas para poder analizar las consecuencias del propio saber que detentan. Especialmente, existen serios límites para predecir el futuro y las reacciones de los otros como respuesta a esas decisiones.

Se asume que los actores tienen una racionalidad limitada, en donde los procesos de decisión son complejos, y en el que interactúan actores que buscan obtener sus objetivos, en ambientes de incertidumbre, riesgo y conflicto, donde las instituciones expresadas en organizaciones, cultura, normas y disposiciones formales e informales inciden en los procesos de formulación y decisión de políticas públicas (Meny y Thoenig, 1992).

\section{c) El incrementalismo}

Como crítica a la concepción racionalista surge la corriente denominada incrementalista, la cual da cuenta de la participación real de diversos actores y la necesidad de romper con los procesos racionalistas de formulación de política pública. Esta perspectiva de la toma de decisiones fue planteada por Lindblom (1994), quien parte del supuesto que el modelo racional es poco útil para la formulación de la política pública. En el modelo desarrollado por Lindblom (1994) el proceso de elaboración de la política es un proceso de aproximación sucesiva a un objetivo en que lo deseado cambia y es reconsiderado de forma continua en el tiempo. A diferencia del modelo racional, las políticas no llegan a finalizarse nunca, porque él mismo señala que las políticas están en constante revisión y modificación.

Mientras que los modelos racionalistas suelen otorgar a los responsables de la toma de decisiones un alto grado de control de la situación. El incrementalismo plantea que se tiene poco control sobre el entorno (Aguilar, 1994); y sugiere fijar la atención en otro nivel al que Lindblom (1994) denomina valores marginales, el autor propone revisar la decisión pública en función de identificar los pequeños problemas que la afectan, y que ameritan ser modificados para mejorarla, y no centrarse en los grandes temas. 
Subirats (1989) plantea que en este modelo de decisión, el decisor goza de independencia partidista y su criterio de selección es el compromiso. Los administradores (funcionarios) -señala Lindblom (1994)- "han de elegir únicamente entre las diversas políticas alternativas que ofrecen diferentes combinaciones marginales de valores" (Lindblom, 1994: 231). Según éste autor el análisis estratégico es el que potencia las capacidades de decisión pues permite analizar con detalle y profundidad la relación real que existe entre los medios y los fines de una organización. Una vez delimitado su campo de acción, el funcionario se ocupará no de elegir la mejor de las políticas posibles, puesto que no es factible llevarlo a cabo, sino aquellas que ofrezcan un incremento de valor marginal respecto a las políticas anteriores o a la política alternativa. Para ello se ofrecen comparaciones entre las ventajas que ofrece una en relación a otra política.

El incrementalismo como modelo de toma de decisiones no escapa a críticas. Dror (1994), fue realizando propuestas para aumentar la racionalidad, a partir de críticas al incrementalismo que surgió como respuesta al racionalismo. Así señala que el incrementalismo no es un método adecuado para la toma de decisiones a menos que se cumplan simultáneamente tres condiciones: a) los resultados de las políticas actuales deben ser satisfactorios tanto para quienes toman las decisiones como para quienes son afectados por ellas, de manera que se pueda considerar que los cambios marginales proporcionan resultados aceptables; b) no debe variar mucho la naturaleza de los problemas $\mathrm{y}$; $\mathrm{c}$ ) debe permanecer constante la disponibilidad de medios para atenderlos. Cuando los resultados de las políticas anteriores no son deseables, es preferible asumir los riesgos que implica tomar decisiones radicalmente nuevas.

Etzioni (1994) plantea críticas tanto normativas como conceptuales y empíricas al incrementalismo. Las críticas normativas son dos: a) las decisiones tomadas con base en el incrementalismo reflejan necesariamente los intereses de los más poderosos, por lo que las demandas de los no privilegiados y de los políticamente desorganizados son sub-representadas; b) tiende a negar las innovaciones sociales básicas en virtud de que su visión es inmediata y busca sólo variaciones limitadas con respecto a las políticas del pasado. Las críticas desde el punto de vista conceptual y empírico, refieren que el incrementalismo reconoce que al concentrarse en las decisiones marginales no toma en cuenta las decisiones fundamentales.

El incrementalismo permite mantener constante el tema central de la discusión y adaptar medios y fines de acción más operativos. Para la ejecución de la planificación incremental se necesitan el desarrollo de un sistema de información que permita el seguimiento, la evaluación periódica de las medidas tomadas y el establecimiento de canales efectivos de comunicación y negociación.

Aun cuando los modelos de decisión han sido los tradicionalmente utilizados, la dinámica de los Estados ha conllevado al fomento de otros modelos de decisión que se desprenden de la acción de la gente organizada y con capacidad de participación ciudadana, aptos de sugerir problemas, de generar influencia en la inclusión en la agenda, de proponer alternativas de solución y propiciar la toma de 
decisiones. Compartimos con Arellano (1996), que la propuesta racional es insuficiente, parcial y generadora de alternativas, por lo que se hace necesario considerar otros modelos de decisión de las políticas públicas.

\section{d) Racionalidad Imperfecta}

Aludir a una racionalidad imperfecta de la razón no niega la validez de la racionalidad sino solamente la imposibilidad de una sola visión abarcadora, clara y precisa del mundo. La racionalidad imperfecta se erige sobre la participación de los ciudadanos en el proceso de formulación de la política pública, como una acción colectiva con racionalidades diferentes (Arellano, 1996); es decir, que no es contraria o excluyente de un proceso de racionalidad, solo que la postura de Arellano, involucra una diversidad de actores en la etapa de formulación, muy distinto a lo que señala la racionalidad como tal, vista sólo desde el Estado. Se constituye en el extremo opuesto de la racionalidad absoluta.

La racionalidad imperfecta, en la cual se trabaja con problemas sociales que imponen una relación Estado-Sociedad, donde toda relación social es resultado de la interacción de múltiples racionalidades distintas, individuales o de grupos. Esta racionalidad alude a la acción colectiva, la cual ha significado un cambio contundente en el análisis de la participación en política y la formación de grupos... la lógica de la acción colectiva es un punto neurálgico en el análisis de la participación de distintos actores (Villaveces, 2009).

Arellano (1996), refiere que las problemáticas sociales son preocupaciones menos adecuadamente entendidas y me- surables que cualquier problema técnico. Los problemas sustantivos de la política pública, problemas de acción colectiva y de participación ciudadana, requieren la incorporación de un juego diferente a la tradicional racionalidad, y la cuestión "...no es fácil, sobre todo porque el enfoque racional es la versión extrema de los principios elementales de la razón" (Arellano, 1996: 322).

Esta mirada amplía la forma tradicional de ver las políticas públicas desde el Estado como actor principal de toma de decisiones. "La incorporación metodológica de la acción colectiva al análisis de política pública es una respuesta tanto a la posición ortodoxa de las políticas públicas centradas en el Estado en una dirección top-down, que dejaba aislado a los demás actores de la sociedad; como a la visión reduccionista que describe las decisiones de los actores en un mundo de individuos racionales, optimizadores y en competencia continua, lo cual no corresponde a un panorama de la vida real, donde, además de situaciones de racionalidad individual, encontramos multitud de casos de cooperación que no siempre se caracterizan por una lógica racional" (Villaveces, 2009: 8).

En este sentido, la orientación en las preferencias y determinación de objetivos, debido a la participación de varios actores mediante una acción conlleva al desarrollo de redes de política (s), lo que representa una nueva modalidad de coordinación de las políticas, que se distingue, como afirma Fleury (2002) de los modelos anteriores. Una forma de acción colectiva se puede visualizar a través de la existencia de redes o estructuras multicéntricas, que comprenden diferentes ac- 
tores y organizaciones vinculados entre sí a partir del establecimiento y mantenimiento de objetivos comunes que conforman una dinámica de gestión compatible y adecuada. Estas redes establecen una nueva relación Estado-Sociedad, originando de esta manera una transformación en el sistema político, donde la participación de la población en la formulación de las políticas públicas crea condiciones propicias para el desarrollo de la ciudadanía y la emancipación de los sectores más marginados de la población y, al mismo tiempo, transforma las estructuras autoritarias del Estado y genera formas de gestión pública conjunta.

Esta gestión conjunta, implica obligatoriamente la participación ciudadana, de manera corresponsable y consciente de las personas a involucrarse de manera individual o colectiva en la formulación de las políticas públicas, de tal forma que permitan la consolidación de un Estado democrático, social de derecho y de justicia (Grimau, 2010). Es la participación amplia que se traduce en su organización desde su diversidad para tomar decisiones y ejercer el control social ciudadano permanente a través de los diversos instrumentos de participación creados para tal fin en el ejercicio intransferible de su soberanía (Grimau, 2010).

Esto conduce a identificar el poder del pueblo organizado, en las más diversas y disímiles formas de participación, para la toma de decisiones en todos sus ámbitos: político, económico, social, ambiental, organizativo, internacional y otros, para el ejercicio pleno de su soberanía (Grimau, 2010). El enfoque de redes como una alternativa innovadora de coordinación en las políticas públicas ex- plica las relaciones entre diversos actores de la sociedad en el proceso de formulación de políticas públicas. Es una forma de dar cuenta de la participación de diversos actores y demostrar que los procesos de formulación de políticas públicas no son exclusivos de los expertos.

Señala Fleury (2002: 226) que "La multiplicidad de actores sociales que influyen en el proceso político, en la decisión..., apunta al florecimiento de una sociedad multicéntrica, en que se organizan distintos núcleos de unión que, a su vez, tienden a alterar los nexos verticales entre el Estado y la sociedad, basados en la reglamentación y subordinación, con orientación hacia las relaciones más horizontales y que favorecen la diversidad y el diálogo".

En tal sentido, Klijn (1998: 9), refiere que "El interés reciente en el concepto de redes de políticas públicas puede verse como un intento de 'contextualizar' el enfoque de proceso. No sólo la formulación de política tiene lugar en escenarios en los que hay muchos actores y ambigüedad en cuanto a las preferencias, información y estrategias tomadas, sino que también sucede en ciertas redes inter organizacionales de naturaleza más duradera... Los problemas, los actores y las percepciones no son elementos fortuitos en el proceso de políticas, sino que están conectados con la red... en la que esos procesos ocurren".

El análisis de redes explica: quien hace la política y cómo la hace. Las redes son estructuras en los que operan agentes que interpretan, construyen y reconstruyen las redes, éstas no son permanentes. Ellas cambian parcialmente en función de las decisiones estratégicas de los agentes 
dentro de la estructura, y estas decisiones estratégicas representan respuestas tanto a factores endógenos como exógenos. La red es estructural porque prescribe los asuntos que son discutidos, como deben ser tratados, posee un conjunto distintivo de reglas y contiene imperativos organizacionales (Zurbriggen, 2003).

Con el enfoque de redes se trata de superar la concepción racionalista en el proceso de formulación de la política pública, ya que en la misma interviene una pluralidad de actores, con lo cual se permite una mayor movilización de recursos y se garantiza la diversidad de opiniones sobre el problema (Fleury, 2002).

El enfoque de redes, describe de forma convincente la realidad del “....proceso de elaboración de políticas públicas, pero tiene una capacidad explicativa todavía débil. Es un instrumento útil que sirve para identificar las variables explicativas a tener en cuenta en el análisis, definir conceptos y categorizar la información..." (Cháques, 2004: 35), es decir, es un enfoque que se usa fundamentalmente para explicar el proceso de formulación de políticas.

La novedad del enfoque de redes reside en que cada política pública genera su propio entramado de actores, se estructura a través de canales institucionalizados propios y sigue una dinámica distinta en la que puede predominar el conflicto o el consenso en torno a los objetivos y estrategias a seguir a lo largo del tiempo (Cháques, 2004).
Cháques (2004) indica que el enfoque de redes niega la posibilidad de utilizar un método único para explicar el proceso de elaboración de las políticas públicas. Se basa en la idea de diversidad de las relaciones Estado-Sociedad y la necesidad de desagregación del análisis para comprender de forma más completa las políticas públicas.

Bajo el enfoque de redes en una situación en la que hay muchos actores con estrategias distintas y gran variedad de objetivos, los actores no pueden saber con antelación cuáles resultados ocurrirán probablemente y cuáles objetivos encontrarán en el proceso. Tienen que aprender esto de forma parcial durante el mismo proceso. Esto significa que la interacción estratégica es una característica importante de los procesos en las redes (Cháques, 2004).

La participación de varios actores sociales y políticos, que manejan diversos instrumentos de participación, solo intervienen para proponer diversos problemas, sugerir alternativas de solución a través de procesos de coalición para apoyar y defender la decisión y para hacer esto no se cuenta con un método único para la formulación de políticas públicas. Estos enfoques propician un proceso de formulación descentralizada y horizontal que evidencia una nueva forma o método de hacer políticas públicas.

El proceso de formulación de políticas públicas, conlleva como se menciona con anterioridad a reflejar la formalidad en la que se presentan las políticas y el contenido al que da lugar dicha política. 


\section{Políticas públicas: de la formulación a su formalidad y contenido}

Ochoa (2007), considera que del proceso de formulación de la política pública emana su producto principal el cual es la política formal, que se constituye en el curso de acción o regla que debe seguirse en la implementación, y la cual define la orientación de la política de la dirección del Estado. Considera la autora en referencia, que en el proceso de formulación participan diferentes y diversos actores visibles e invisibles, unos con mayor o menor poder que otros y varían de acuerdo a la realidad objeto de atención.

Esta política formal se plasma en diversos documentos que imponen las reglas, desde los más generales y de principios hasta los más específicos y coyunturales. Siendo los mismos las constituciones, leyes, reglamentos, decretos y programas, y la formalidad de las decisiones se refleja en la publicación en gaceta, lo cual se constituye en un acto válido para que cualquier política se considere válida (Ochoa, 2007; Kelly, 2004: 71).

En estos documentos oficiales se refleja el contenido de la política, el cual está asociado a la orientación que la “...decisión de una autoridad, adopta dentro de su campo legítimo de jurisdicción y conforme a procedimientos legalmente establecidos, vinculantes para todos los ciudadanos..." (Aguilar, 1994: 22), no es un fenómeno que se autodefine, sino una categoría analítica. Es el análisis el que identifica su contenido, no los dichos del decisor de la política ni las piezas del legislador de la administración...La política existe no por intuición sino por interroga- ción de los fenómenos políticos" (Heclo, 1972 citado por Aguilar, 1994: 22).

Para Subirats y Gomá (1998), y Font (2001), el contenido es la dimensión sustantiva de toda política pública, el cual puede ser identificado a través del(os) objetivo(s): entendido como el fin o intento que persigue la política, del(os) principio(s): norma o idea fundamental que debe regir la política en cuanto organización, funcionamiento y acción. Otro elemento del contenido son los mecanismo(s): medios a través de los cuales se estructura la política. Además de los elementos planteados por estos autores, se considera importante agregar a los beneficiarios que persigue favorecer o cubrir la política pública diseñada, es decir a las personas nacidas o legalmente residenciadas en una región determinada.

Ahora bien, indica Lowi en 1972 que el contenido de la política pública varía de acuerdo a criterios de coerción; y por el impacto de costo y beneficio que los grupos de interés esperan de una política determinada, es una clasificación de política según la intencionalidad de la misma; en este sentido, la política pueden ser:

a) Distributiva: Es una arena relativamente pacífica, se caracteriza por cuestiones no rivales que pueden ser tratadas con recursos públicos divisibles, en ésta se hacen acuerdos entre los demandantes y los grupos de interés. Distribuyen privilegios, beneficios a sectores/segmentos de la sociedad, o a espacios/territorios concretos.

b) Regulatoria: Es una arena de conflicto y negociación entre los grupos de poder, es una arena turbulenta, de intereses exclusivos y contrapuestos de grupos que se ven obligados a coali- 
ciones o transacciones de recíproca concesión donde la solución no favorece a las partes por igual, hay beneficiarios y afectados. Tienen como objetivo dictar normas que disciplinen, regulen y limiten una actividad.

c) Redistributiva: Es la más audaz y radical, aborda cuestiones de las relaciones de propiedad, poder y prestigio social establecido. Es la arena más tensa y frontalmente conflictiva, muy semejante a la clásica lucha de clases del marxismo, aquí las transacciones son imposibles o con poco resultados, debido a que puede afectar a grupos o sectores de la población. Tiene que ver con el modo en que se distribuye la riqueza, la renta nacional. Generan beneficios a amplios sectores de la sociedad.

d) Constituyente: Modifica las reglas del juego político y se encuentran relacionadas con modificaciones del régimen político y/o administrativo, puesto que amplían o disminuyen la jurisdicción gubernamental, alterando en mayor o menor grado el ejercicio de los derechos de los ciudadanos, restringiendo o aumentando las opciones de acción privada o pública, se crea o modifica una institución.

La clasificación planteada por Lowi (1972), conduce a la identificación del contenido de la política diseñada para un determinado sector $y$, por ende, precisa el juego político en la estructura decisional. Por lo que la relación entre contenido y estructura de la decisión es algo relativamente insoluble. Este contenido, marca la orientación que emana de un proceso de formulación de una política pública.

\section{Reflexiones finales}

El proceso de formulación de las políticas públicas se constituye en un esquema metodológico que conjuga el proceso de la elaboración a partir de un análisis sistematizado y coherente, guiado por una batería de interrogantes que no son limitativas ni se agotan en un número determinado. Esta etapa de las políticas se constituye en eje fundamental para el análisis de todo el proceso de formación de las políticas, si no existe una adecuada definición del asunto o problema a atacar el proceso sucesivo sin duda tendrá fallas técnico operativas que no permitirá obtener resultados positivos para contrarrestar los obstáculos manifestados en los distintos y diferentes asuntos públicos.

Para el estudio de los procesos de formulación de las políticas públicas, se hace necesario considerar diversos enfoques teóricos para la toma de decisiones, en los cuales se conjugue el papel del Estado y su relación con la sociedad, de tal manera de establecer un análisis objetivo y equilibrado. En este sentido, el análisis bajo los modelos racionales de decisiones, reflejan que la elaboración de las políticas públicas se caracteriza por ser responsabilidad del Estado.

Pero, bajo otros enfoque de decisión como la racionalidad imperfecta 0 enfoque de redes, se apunta hacia la participación de diferentes y diversos actores en el proceso de formulación de la política pública, con lo que se establece una nueva relación Estado -Sociedad, y en la que se aúpa la configuración de la política desde una lógica contraria a la tradicional, es decir, se pasa de una vieja concepción de arriba hacia abajo a una de 
abajo hacia arriba, lo que demuestra que el surgimiento de la intermediación de interés, como un aspecto neurálgico para analizar las diversas formas de articulación Estado y Sociedad en las diferentes áreas de políticas públicas, lo cual emerge como consecuencia de los límites de los modelos tradicionales de analizar las políticas públicas, a pesar de no establecer un método de análisis determinado, pues este tipo de modelo de decisión considera que cada política tiene su propia lógica de análisis y de participación de actores según el tema de atención.

La formulación de las políticas públicas, dan paso no solo a su elaboración, sino que a partir de dicho proceso surge la formalidad de las mismas, las cuales aparecen reflejadas en documentos oficiales y publicados en gacetas $u$ otros mecanismos legislativos, y es de esta subfase surge la dimensión sustantiva que persigue la política pública elaborada.

\section{Referencias Bibliográficas}

Aguilar, Luis (1994). "Estudio Introductorio”. La Hechura de las Políticas. Miguel Ángel Porrúa. Grupo Editorial. México

Aguilar, Luis (2000). "Estudio introductorio". Problemas, Públicos y agenda de gobierno. La implementación de las políticas. Editorial Miguel Ángel Porrúa, México

Aguilar Villanueva, Luis F. (2003). La implementación de las políticas públicas. Tercera edición, Editorial Miguel Ángel Porrúa. México. pp. 28-316

Aktouf, Omar (1998). La administración entre tradición y renovación. Artes Gráficas del Valle. Colombia

Álvarez, Ángel (1992). Análisis de políticas públicas, CLAD, Caracas.
Arellano Gault, David (1996). "Política Pública, racionalidad imperfecta e irracionalidad. Hacia una perspectiva diferente". En Gestión y Política Pública. Vol. V. No. 2. Pp. 319-347. México

Chaqués, Laura (2004). Redes de Políticas públicas. Centro de Investigaciones Sociológicas. Siglo XXI, España.

Dror, Yehezkel (1994). "Salir del paso ¿ciencia o inercia?". En Aguilar Villanueva, Luis.(1994). La Hechura de las Políticas. Editorial Miguel Ángel Porrúa. México

Dunn, William (1994). Public policy analysis: An introduction. 2nd edition. Englewood Cliff, NJ.: Prentice Hall.

Elster, John (1985). "Rationality, Morality and Collective Action", En Ethics, 96, 136-155.

Etzioni, Amitai (1994). "La exploración combinada: un tercer enfoque de la toma de decisiones". En Aguilar Villanueva, Luís (1994). La Hechura de las Políticas. Editorial Miguel Ángel Porrúa. México.

Fleury, Sonia (2002). "El desafío de la gestión de las redes de políticas". En Revista Instituciones y Desarrollo, № 12-13 (2002) págs. 221-247. Institut Internacional de Governabilitat de Catalunya, Barcelona, España. En http://www.iigov.org, fecha de consulta 25/09/2010

Fleury, Sonia (2004). "Ciudadanías, Exclusión y democracia". En Nueva Sociedad. No. 193. Caracas, pp. 62-75

Font, Joan (2001). Ciudadanos y decisiones públicas, Editorial Ariel, Barcelona.

Forester, John (1994). "La racionalidad limitada y la política de Salir del paso". En Aguijar Villanueva, Luis (1994). La Hechura de las Políticas. Editorial Miguel Angel Porrúa. México

Grau, Mireia (2002). "El estudio de las políticas públicas: enfoques y metodologías de análisis". En GRAU, Mireia y MATEOS, Aracelis (Edit). Análisis de Po- 
líticas Públicas en España: enfoques y casos. Colección Ciencia Política. Edita Tirant Lo Blanch. España

Grimau, Roso (2010). VENEZUELA avanza construyendo PODER POPULAR hacia el SOCIALISMO del Siglo XXI. En http://rosogrimau.blogspot.com/20 10/02/concepto-de-poder-popularpara-el.html,fecha de consulta 23 de noviembre de 2010

Kelly, Janet (2004). Políticas Públicas en América Latina. Teoría y Práctica. Ediciones IESA. Caracas.

Kingdon, John (1984). Agendas, alternatives and public policies, Little, Boston.

Klijn, Elko (1998). "Policy Networks: An Overview". En Kickert, W. J. M. \& Koppenjan, J.F.. (eds). Managing Complex Networks. Sage, London. Traducido por Mariángela Petrizzo. En revista-redes.rediris.es/webredes/ textos/Complex.pdf, fecha de consulta $12-05-2010$

Lapierre, Jean-William (1976). El análisis de los sistemas políticos. BarcelonaEspaña.

Lindblom, Charles (1994). La ciencia de salir del paso. En Aguilar, Luis La hechura de las políticas. Miguel Ángel Porúa. México.

Losada Trabada, Antonio (2003). "Entre la ciencia política básica y la ciencia política aplicada; de la política a las políticas, del análisis a la gestión". En Revista de Investigación Políticas e Sociológicas, Vol. 2, Nos: 1-2, pp. 63-81

Lowi, Theodore (1972). "Four Systems of Policy, Politics and Choice". En Public Administration Review, Vol. 32, No. 4, pp. 298-310.

Meny, Ives y Thoenig, Jean (1992). Las políticas públicas. Ariel. Barcelona, España.

Miller, Hugh (1994). "Post-progressive Public Administration: lessons from Policy Networks". En Public Administration Review, Vol. 54 No. 4. http:// aas.sa- gepub.com/content/37/4/504 .full.pdf, fecha de consulta 24-06-2004

Muller, Pierre (2006). Las Políticas Públicas. 2da edición. Traductores Jean-Francois y Salazar, Carlos. Universidad Externado de Colombia. Colombia.

Ochoa, Haydée (2007). "Concepción Racionalista de las Políticas Públicas". Presentación para el Seminario de Gestión y Políticas Públicas. UNERMB, Cabimas, Venezuela.

Roth, André (2004). Políticas Públicas: Formulación, Implementación y Evaluación. Ediciones Aurora, Bogotá, Colombia

Roth, André (2007). Políticas Públicas: Formulación, Implementación y Evaluación. Ediciones Aurora, Bogotá, Colombia

Ruiz, Carlos (1996). Manual para la elaboración de políticas públicas. Plaza Valdés y universidad lberoamericana. México.

Simon, Herbert (1986). "De la racionalidad sustantiva a la procesal", en Hahn, Frank y Martin Hollis, Filosofía y teoría económica, FCE, México, pp. 130-171.

Subirats, Joan (1989). Análisis de Políticas Públicas y eficiencia de la Administración. Instituto nacional de Administración Pública. Madrid, España.

Subirats, Joan y Gomá, Ricard (1998). Políticas Públicas: Hacia la renovación del instrumental de análisis“. En GOMÁ, Ricard y SUBIRATS, Joan (coords.). Políticas Públicas en España. Contenidos, Redes de actores y niveles de Gobierno. Ariel Barcelona.

Therborn, Goran (1989). Las teorías contemporáneas del estado. Valdes hermanos Editores. Venezuela

Urdaneta, Joheni (2010). Política Nacional de Transporte Urbano en Venezuela 1989-2008. Tesis para optar al título de Doctora en Ciencias Sociales, 
mención Gerencia. Maracaibo. Universidad del Zulia. Facultad de Ciencias Económicas y Sociales. División de Estudios para Graduados. Maracaibo. Venezuela.

Villaveces Niño, Juanita (2009). "Acción colectiva y el proceso de la política pública”. Revista Opera No. 9, pp. 7-22, Universidad Externado de Colombia. En http://redalyc.uaemex.mx/src/ini-
cio/ArtPdfRed.jsp?iCve $=6751500700$

2, fecha de consulta 4-10-2009

Zurbriggen, Cristina (2003). "Las redes de políticas públicas. Una revisión teórica". En http://www.iigov.org/documentos, fecha de consulta 23 de julio de 2009

Peterson, John (2003). Policy Networks. Political Science Series. Viena: Institute for Advanced Studies. En www.ihs.ac.at/publications/pol/pw_90.pdf 